\title{
Catalyses by Polymer Complexes. VI. Catalysis of Proton Abstraction from $a$-Ketols by Hydroxamate Ions Bound to Polymer Micelles*
}

\author{
Seiji Shinkai and Toyoki Kunitake \\ Department of Organic Synthesis, Faculty of Engineering, \\ Kyushu University, Fukuoka 812, Japan.
}

(Received March 29, 1977)

\begin{abstract}
Hydroxamate anions bound to partially laurylated poly(2-ethyl-1vinylimidazole) were found to serve as excellent catalysis for proton abstraction from $\alpha$-ketols. Proton removal from benzoin by $N$-methylmyristohydroxamate anion in the presence of the polymer (lauryl group content, $29 \mathrm{~mol} \%$ ) was $2.1 \times 10^{4}$ times faster than the corresponding reaction in the absence of the polymer, and 330 times faster than that by hydroxide ion. The maximum catalytic efficiency existed at $c a$. 30-mol $\%$ lauryl group content. Addition of $\mathrm{KCl}$ and ethanol significantly suppressed the reaction. Thus, hydroxamate-polymer complexes would act generally as excellent base catalysts for proton abstraction to form the carbanion intermediates.

KEY WORD Polymer Micelle / Proton Abstraction / Hydroxamic Acid / Hydrophobic Environment / Polymer Complex / Activation of Anion /
\end{abstract}

Previously we pointed out that some anionic nucleophiles are greatly activated in the presence of cationic micelles, ${ }^{1}$ cationic polysoaps, ${ }^{2,3}$ and other cationic hydrophobic aggregates. ${ }^{4}$ The large activation of these anionic species appears to be explained by the formation of hydrophobic, desolvated ion pairs. This finding seems of great importance in connection with the enzymatic catalysis, since the active sites of enzymes are situated at the hydrophobic pocket and the anionic function plays a crucial catalytic role. ${ }^{5}$

Proton abstraction from carbon acids is a representative, base-catalyzed reaction, and is associated with the enzymatically important reactions such as racemization, enolization, and condensation. We recently demonstrated that the rate-determining step for the oxidation of $\alpha$-ketols by oxygen, dichlorophenolindophenol and lumiflavin (vitamin $\mathbf{B}_{2}$ analogue) is basecatalyzed proton abstraction process (eq 1$)^{6}$ and

* Contribution No. 415 from this department. Presented in part at the 25th Annual Meeting of the Society of Polymer Science, Japan, Tokyo, May, 1976. Part V. S. Shinkai, K. Tou, and T. Kunitake, Polym. J., 9, 381 (1977). that the reaction is facilitaded by the use of the desolvated ion pair as a base. ${ }^{7}$

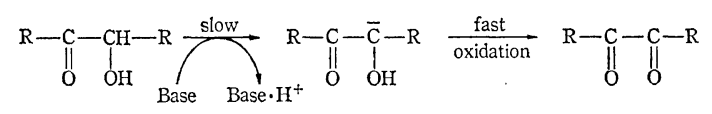

We should like to describe here the proton abstraction reaction from benzoin and furoin catalyzed by hydroxamate anions bound to cationic polysoaps. The object of the present study is to assess the hydrophobic and electrostatic influences on the basicity of bound hydroxamate anions. Hydroxamic acids employed are $N$-methylmyristohydroxamic acid (MMHA), $\mathrm{N}$-laurylbenzohydroxamic acid (LBHA), and $\mathrm{N}$ benzylbenzohydroxamic acid (BBHA). Polymer micelles were prepared from poly(2-ethyl-1-vinylimidazole) by quaternization with alkyl bromides.

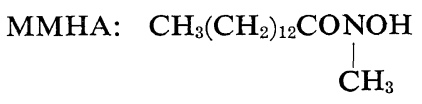

LBHA:

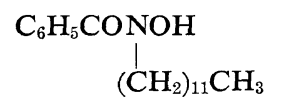




\section{S. Shinkai and T. Kunitake}

BBHA:<smiles>O=[W](O)C(Cc1ccccc1)c1ccccc1</smiles>

Polysoap:

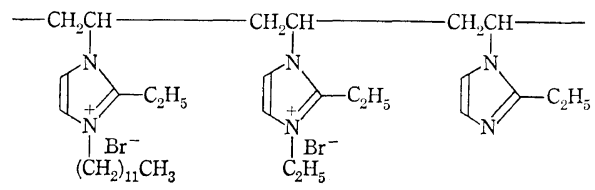

\section{EXPERIMENTAL}

\section{Materials}

The preparation of hydroxamic acids and polysoaps have been described elsewhere. ${ }^{2,8,9}$ The composition of polysoaps used is listed in Table I. Benzoin and furoin were recrystallized from ethanol-water before use.

Table I. Composition of polysoaps

\begin{tabular}{lccc}
\hline Polysoap & $\begin{array}{c}\text { Lauryl } \\
\text { group, } \\
\text { mol \% }\end{array}$ & $\begin{array}{c}\text { Ethyl } \\
\text { group, } \\
\text { mol \% }\end{array}$ & $\begin{array}{c}\text { Unquaternized } \\
\text { unit, } \\
\text { mol \% }\end{array}$ \\
\hline L-9 & 9 & 83 & 8 \\
L-29 & 29 & 67 & 4 \\
L-41 & 41 & 49 & 10 \\
\hline
\end{tabular}

\section{Kinetics}

Kinetic measurements were carried out at $30^{\circ} \mathrm{C}$ in 3-vol $\%$ aqueous ethanol by using modified Thunberg cuvettes unless stated otherwise. Buffer solutions of $\mathrm{pH} 11.4$ were prepared from $\mathrm{KOH}$ and $\mathrm{O}_{2}$-saturated water. The hydroxamic acids (MMHA, LBHA, and BBHA) are fully ionized at this $\mathrm{pH}$. Further details of the procedure were described in ref 7 .

The rate-determining step of the oxidation of $\alpha$-ketols to the corresponding diketones is basecatalyzed proton abstraction, and the overall rate is independent of the nature and concentration of the oxidizing agent. ${ }^{6,7}$ In the present investigation, molecular oxygen saturated in buffer solutions was used as the oxidizing agent.

The reactions were observed on a Hitachi 124 spectrophotometer at $280 \mathrm{~nm}$ for benzoin and $310 \mathrm{~nm}$ for furoin. The difference in absorbance between $\alpha$-ketols and diketones was greatest at these wavelengths. The pseudo first-order rate constants $\left(k_{\mathrm{obs}}\right)$ were determined from the linear plots of the first-order equation. The secondorder rate constant for the hydroxide catalysis $\left(k_{\mathrm{OH}}\right)$ is given by eq $2 .^{7}$ The oxidation rates in the case of the hydroxamate catalysis are also first order in the hydroxamate anion, and the second-order rate constant $\left(k_{\mathrm{HA}}\right)$ is expressed by eq 3

$$
\begin{gathered}
k_{\mathrm{OH}}=k_{\mathrm{obs}} /\left[\mathrm{OH}^{-}\right] \\
k_{\mathrm{HA}}=\left(k_{\mathrm{obs}}-k_{\mathrm{spont}}\right) /[\text { hydroxamate }]
\end{gathered}
$$

where $k_{\text {spont }}$ denotes pseudo first-order rate constants in the absence of the hydroxamate ion.

\section{RESULTS AND DISCUSSION}

\section{Hydroxide Catalysis}

The proton abstraction reaction from benzoin

\begin{tabular}{|c|c|c|c|c|c|}
\hline \multicolumn{2}{|c|}{ Polymer } & \multirow{2}{*}{$\begin{array}{c}k_{\mathrm{OH}}, \\
M^{-1} \mathrm{~s}^{-1}\end{array}$} & \multicolumn{3}{|c|}{$k_{\mathrm{HA}}, M^{-1} \mathrm{~s}^{-1}$} \\
\hline & $\mathrm{m} M$ & & MMHA & LBHA & BBHA \\
\hline None & & 0.081 & - & - & 0.0013 \\
\hline L-9 & 2.06 & 0.154 & 7.74 & 1.21 & - \\
\hline L-29 & 1.49 & 0.480 & 27.2 & 27.1 & - \\
\hline $\mathrm{L}-29^{\mathrm{b}}$ & 1.49 & 0.128 & 19.6 & 19.0 & - \\
\hline $\mathrm{L}-29^{\mathrm{c}}$ & 1.49 & 0.175 & 3.58 & 3.82 & - \\
\hline L-41 & 1.43 & 0.564 & 22.8 & 19.0 & - \\
\hline $\mathrm{CTAB}^{\mathrm{d}}$ & 5.00 & 0.569 & 4.53 & - & 0.489 \\
\hline
\end{tabular}

Table II. Proton abstraction rates from benzoina

a $30^{\circ} \mathrm{C}, 3-\mathrm{vol} \%$ ethanol. The only inorganic substant was $\mathrm{KOH}$.

b $\mu=0.5$ with $\mathrm{KCl}$.

c 25.7 -vol \% ethanol.

a Cetyltrimethylammonium bromide: cited from ref 7 .

Table III. Proton abstraction rates from furoin ${ }^{\mathrm{a}}$

\begin{tabular}{lccccc}
\hline \multicolumn{2}{c}{ Polymer } & \multirow{2}{*}{$k_{\mathrm{OH}}}$, & \multicolumn{3}{c}{$k_{\mathrm{HA}}, M^{-1} \mathrm{~s}^{-1}$} \\
\cline { 1 - 3 } \cline { 5 - 6 } & $\mathrm{m} M$ & $M^{-1} \mathrm{~s}^{-1}$ & MMHA & LBHA & BBHA \\
\hline None & & 2.80 & - & - & 0.056 \\
L-9 & 2.06 & 3.50 & 51.6 & 20.4 & - \\
L-29 & 1.49 & 4.20 & 176 & 168 & - \\
L-41 & 1.43 & 4.20 & 138 & 75.9 & - \\
CTAB $^{\text {b }}$ & 5.00 & 4.00 & 23.1 & - & - \\
\hline
\end{tabular}

a $30^{\circ} \mathrm{C}, 3-\mathrm{vol} \%$ ethanol. The only inorganic substance used was $\mathrm{KOH}$.

b Cetyltrimethylammonium bromide; cited from ref 7 . 
and furoin was examined in the absence and presence of polymer micelles: L-9 (lauryl group content, $9 \mathrm{~mol} \%)$, L-29 (29 $\mathrm{mol} \%)$, and L-41 $(41 \mathrm{~mol} \%)$. Typical rate constants for the hydroxide catalysis are summarized in Tables II and III.

In the absence of these polymer micelles, the proton abstraction rate from furoin was 35 times faster than that from benzoin. This difference reflects a greater acidity of furoin (a carbon acid) than benzoin. When polymer micelles were added, the oxidation rate increased with increasing polymer concentrations, and finally reached saturation values (Figure 1). A similar

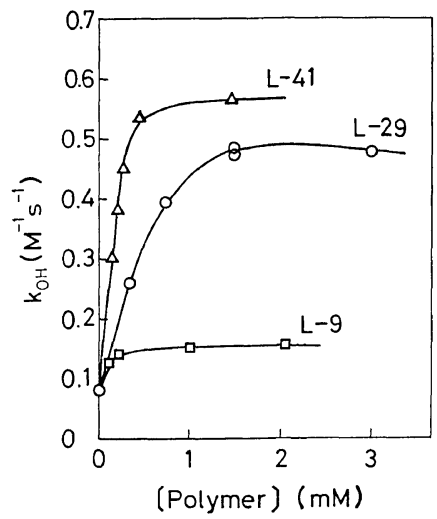

Figure 1. $k_{\mathrm{OH}}$ for proton abstraction from benzoin vs. polymer concentration: [benzoin] $=5.00 \times 10^{-5} \mathrm{M}$, $\mathrm{pH} 11.40$.

trend was observed for furoin substrate. The polymer concentration was calculated based on the total monomer units. L-29 and L-41 were efficient catalysts, and the rate augmentations were $6-7$ and 1.5 for the oxidation of benzoin and furoin, respectively. L-9 was less effective (rate augmentation: 1.9 for benzoin and 1.3 for furoin).

Benzoin was more suceptible to the micellar ${ }^{7}$ and the polymer micellar catalysis than furoin. This is explicable by the favorable partitioning of benzoin in the hydrophobic phase of polymer micelles compared with furoin because of the smaller solubility of benzoin in aqueous media.

\section{Hydroxamate Catalysis}

Pseudo first-order rate constants for the hy- droxamate-catalyzed proton abstraction from benzoin $\left(k_{\mathrm{obs}}-k_{\text {spont }}\right)$ increased linearly with the concentration of MMHA $\left(2 \times 10^{-5} \sim 10^{-4} M\right)$ in the presence of $1.49 \mathrm{mM}$ of L-29. The rate was not affected by further increase in the concentration of L-29. Therefore, the rate is first order with respect to MMHA under the experimental conditions employed ([polymer]》[MMHA]). However, the plots were curved downward at higher MMHA concentrations (above $5 \times 10^{-4} \mathrm{M}$ ), probably because of the changing microenvironment of polymer micelles. Thus, the lowest possible concentration of the hydroxamate anions were used. The second-order rate constants for the hydroxamate catalysis $\left(k_{\mathrm{HA}}\right)$ were calculated according to eq 3 , and summarized in Tables II and III.

Since MMHA and LBHA are virtually insoluble in aqueous media, $k_{\mathrm{HA}}$ for these hydroxamate anions in the absence of the polymer micelle could not be determined, and was approximated by that of BBHA without added polymer micelles.

BBHA in the nonmicellar system is less reactive than hydroxide ion $(1 / 62$ for benzoin and 1/50 for furoin). However, the nucleophilicities of these anions $\left(\mathrm{BBHA}^{-}\right.$and $\left.\mathrm{OH}^{-}\right)$toward $p$ nitrophenyl acetate are similar $\left(9.5 \mathrm{M}^{-1} \mathrm{~s}^{-1}\right.$ at $30^{\circ} \mathrm{C}$ for $\left.\mathrm{BBHA}\right){ }^{10}$ These results can be explained by the fact that the base-catalyzed proton removal from a carbon acid is not subject to the $\alpha$-effect. ${ }^{11}$

The catalytic efficiency of hydroxamate anions in the presence of the polymer micelle is much greater than that of hydroxide ion. As recorded in Table II, MMHA bound to L-29 abstracts proton from benzoin 56 times faster than hydroxide ion with added L-29 polymer, and 330 times faster than hydroxide ion itself. The $k_{\mathrm{HA}}$ value of the MMHA-L-29 system is 21000 fold (for benzoin) and 3300 fold (for furoin) greater than that of the BBHA catalysis in the nonmicellar system. The polymer micelles (L-29 and L-41) enhanced the reactivity of MMHA more effectively than the CTAB (cetyltrimethylammonium bromide) micelle.

\section{Effects of Ionic Strength and Ethanol}

In general, the micelle-catalyzed process is inhibited by added electrolytes. ${ }^{12}$ This is also 


\section{S. Shinkai and T. Kunitake}

true for the catalysis of polymer micelles., ${ }^{2,3}$ Figure 2 shows the effect of ionic strength $(\mathrm{KCl})$ on $k_{\mathrm{OH}}$ and $k_{\mathrm{HA}}$ (benzoin) in the presence of $1.49 \mathrm{mM}$ of L-29. Both of these rate constants decreased with increasing ionic strength. The largest decrease observed was 1.4 fold with the

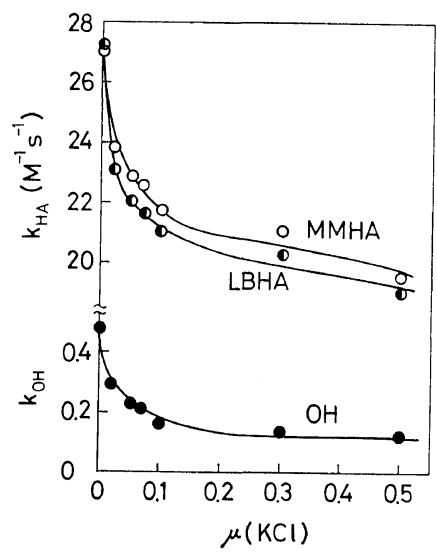

Figure 2. Influence of ionic strength on the hydroxide- and hydroxamate-catalyzed proton abstraction from benzoin: [L-29] $=1.49 \times 10^{-3} M$; [benzoin $]=5.00 \times 10^{-5} \mathrm{M} ;\left[\mathrm{OH}^{-}\right]=3.69 \times 10^{-3} \mathrm{M}(\mathrm{pH}$ 11.40); $[\mathrm{MMHA}]=5.00 \times 10^{-5} M ; \quad[\mathrm{LBHA}]=4.94 \times$ $10^{-5} M$.

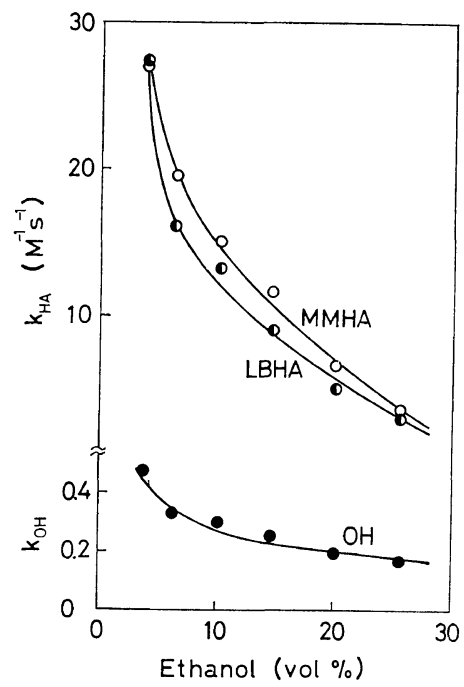

Figure 3. Influence of added ethanol on the hydroxide- and hydroxamate-catalyzed proton abstraction from benzoin: No inorganic substance was added except $\mathrm{KOH}$; other reaction conditions were recorded under Figure 2.
MMHA catalysis and 3.8 fold with the hydroxide catalysis, the differences being relatively small. The rate decreases can be understood in the term of the charge neutralization of the polymer micelle by these added salts. ${ }^{12}$

Addition of alcohol also retarded the reaction (Figure 3). The rate suppression in the hydroxamate catalysis is relatively large ( 7.6 fold with the MMHA catalysis), while the hydroxide catalysis is less influenced (2.7-fold retardation). Addition of organic solvents probably weakens the hydrophobic force of the polymer micelle, so that the hydroxamate catalysis must be closely related to the hydrophobic environment around the reactive anion.

\section{Effect of Lauryl Group Content}

Quaternized polymers possess interesting catalytic behavior in that they combine within a molecule structural characteristics of the conventional micelle (the presence of long alkyl chains) and the polyelectrolyte (the presence of high charge density). ${ }^{9,13,14}$ Many years ago, Strauss, et al. ${ }^{15}$ showed in regard to laurylated poly(vinylpyridines) that the transition to the micellar state occurs at ca. $15-\mathrm{mol} \%$ lauryl substitution. We also demonstrated that strongly hydrophobic microenvironments are formed when the lauryl content is $15 \mathrm{~mol} \%$ or greater, and that bound anionic reagents become greatly activated. ${ }^{2,3,16}$ However, when hydrophobic anions were used in combination with these quaternized polymers, large rate accelerations were observed with much smaller content of the lauryl group. For example, the MMHA anion bound to the L-9 polymer micelle, a polyelectrolyte $^{9}$ is a much better nucleophile toward p-nitrophenyl acetate than by itself. ${ }^{17}$ This is also true in the proton abstraction, as shown by the data of Tables II and III. It is interesting that a significant difference between MMHA and LBHA is seen in the proton abstraction (especially from benzoin) in the presence of L-9. When the formation of hydrophobic region is incomplete, the hydrophobicity of the anion moiety may be reflected by the reaction rate.

Figure 4 is the plot of $k_{\mathrm{HA}}$ against the lauryl group content. The $k_{\mathrm{HA}}$ value is maximal at 29-\% lauryl content for the two hydroxamate anions. Similar maxmai have been observed 
Catalyses by Polymer Complexes. VI.

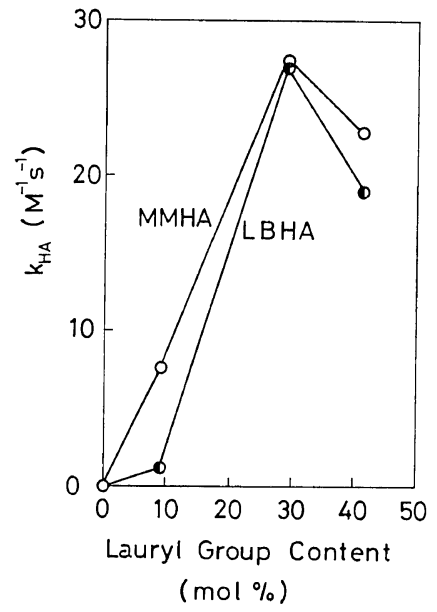

Figure 4. $k_{\mathrm{HA}}$ for proton abstraction from benzoin vs. lauryl group content.

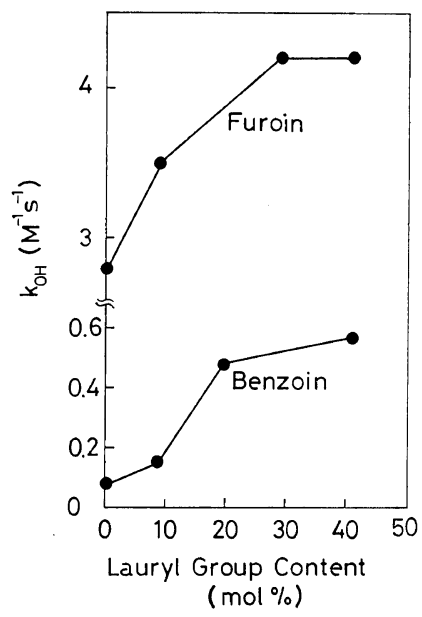

Figure 5. $k_{\mathrm{OH}} v s$. lauryl group content.

for the spectral shift of methyl orange ${ }^{9}$ and for other polysoap-catalyzed reactions. ${ }^{3,9,16}$ These maxima correspond roughly to the position of the viscosity minimum, ${ }^{9}$ that is, the most compact conformation. It is concluded from these results that the polymer micelle is most hydrophobic at ca. 30-\% lauryl substitution and exerts the greatest catalytic effect.

Very similar trends were observed for the influence of the lauryl content on $k_{\mathrm{HA}}$ for furoin substrate: see Table III.

Figure 5 describes the influence of the lauryl group content on the hydroxide-catalyzed proton abstraction rate. The rate enhancements are small and probably attributed to concentration of substrates and hydroxide ion around the polymer micelle. $k_{\mathrm{OH}}$ increases with increasing lauryl group content, and this tendency contrasts with that observed for $k_{\mathrm{HA}}$. Thus, $k_{\mathrm{OH}}$ term may not reflect the conformational change of polymer micelles.

\section{CONCLUSION}

It is now established that cationic polymer micelles can activate the proton abstracting ability of hydroxamate anions at least $10^{4}$ times as much. We have shown that these polymer micelles can enhance the nucleophilic reactivity of the hydroxamate and thiolate anions and the ease of decarboxylation of a carboxylate anion. ${ }^{2,3,15}$ Therefore, organic anions would generally be activated when bound to cationic polymer micelles in water. These results are explained by the concept of "hydrophobic ion pair," which we proposed for the catalytic effect of cationic micelles. ${ }^{1,78}$ It is also expected that the hydroxamate-polymer complex would be used as powerful catalyst for a variety of base-catalyzed reactions in which the carbanion formation is involved in the rate-determining step.

Acknowledgment. We should like to thank Miss R. Ando for her very capable technical assistance.

\section{REFERENCES}

1. T. Kunitake, S. Shinkai, and Y. Okahata, Bull. Chem. Soc. Jpn., 49, 540 (1976).

2. T. Kunitake, S. Shinkai, and S. Hirotsu, Biopolymers, 15, 1143 (1975).

3. S. Shinkai and T. Kunitake, Makromol. Chem., in press.

4. Y. Okahata, R. Ando, and T. Kunitake, $J$. Am. Chem. Soc., 99, 3067 (1977).

5. O. Tsolas and B. L. Horecker, "The Enzymes," 3rd Ed., P. D. Boyer Ed., Vol. 8, Academic Press, New York, N.Y., 1972.

6. S. Shinkai, T. Kunitake, and T. C. Bruice, $J$. Am. Chem. Soc., 96, 7140 (1974).

7. S. Shinkai and T. Kunitake, J. Chem. Soc., Perkin Trans. II, 980 (1976). 
8. T. Kunitake, Y. Okahata, and T. Sakamoto, J. Am. Chem. Soc., 98, 7780 (1976).

9. S. Shinkai and T. Kunitake, Biopolymers, 15, 1129 (1976).

10. T. Kunitake, Y. Okahata, and R. Ando, Bull. Chem. Soc. Jpn., 47, 1509 (1974).

11. R. F. Pratt and T. C. Bruice, J. Org. Chem., 37, 3567 (1972).

12. J. H. Fendler and E. J. Fendler, "Catalysis in Micellar and Macromolecular Systems," Academic Press, New York, N. Y., 1975.
13. T. Rudolfo, J. A. Hamilton, and E. H. Cordes, J. Org. Chem., 39, 2281 (1974).

14. T. Okubo and N. Ise, ibid., 38, 3120 (1973).

15. U. P. Strauss and N. L. Gershfeld, J. Phys. Chem., 58, 747 (1954).

1.6. T. Kunitake, S. Shinkai, and S. Hirotsu, $J$. Org. Chem., 42, 306 (1977).

17. T. Kunitake, S. Shinkai, and S. Hirotsu, unpublished result.

18. S. Shinkai and T. Kunitake, Chem. Lett., 109 (1976). 